\title{
Antifungal Activity of Petroleum Ether and Ethanol Extracts of Moringa Oleifera Seeds
}

\author{
Gamal Rabie ${ }^{1}$, Mohamed Taha ${ }^{2}$, Khaled Youssef ${ }^{3}$ \\ ${ }^{1}$ Professor of Microbiology, Faculty of Science \\ Zagazig University, Egypt \\ ${ }^{2}$ Professor of Microbiology, Faculty of Veterinary Medicine \\ Zagazig University, Egypt \\ ${ }^{3}$ M. Sc. Faculty of Science \\ Zagazig University, Egypt \\ Corresponding author's email: kboozo [AT] yahoo.com
}

\begin{abstract}
The activity of petroleum ether and ethanol extracts of Moringa olifera seeds have antifungal activity on different fungi including dermatophyte, non-dermatophyte molds and yeasts, which isolated from human skin and nail fungal infections. From 70 samples collected from skin and nail infection; 20 dermatophyte isolates, 21 non dermatophyte moulds and 25 yeast were isolated. While macromorphological and micromorphological as well as cultivation on differential media identified all isolated molds (derematophyte and non dermatophye molds), cultivation on corn meal media and chromogenic Candida agar could identified isolated yeasts. EME is having more antifungal activity than PME on all tested fungi.
\end{abstract}

Keywords--- Moringa oleifera (MO) petroleum ether extract (Pe) ethanol extract (Eme) phytochemical dermatophyt non dermatophytes yeast

\section{INTRODUCTION}

The products derived from several herbs and plants, being a source of multifunctional curing agents. About $70-80 \%$ of the world's population relies on herbal and plants medicine to prevent and cure diseases specially in development countries (Ekor 2014). On the other hand about $25 \%$ of the synthesized drugs are manufactured from medicinal plants (Pan et al., 2012). On recent years interests have been generated in the development of plant extracts to control fungal infections. Various plants extracts are found to have antifungal activities with no side effects on human and animals (Tabassum and Vidyasagar 2013). From Those plants which have antifungal effects is Moringa Moringa oliolifera. Moringa olifera is one of the most widely distributed and naturalized species of the monogeneric family Moringaceae (Ramachandran et al 1980). The plant is known for its nutritional and medicinal value. It contains some phytochemicals which make it is good source of antioxidant and antimicrobial substances (Kayode and Afolayan 2015). Concerning antifungal effect it was found that its extracts can affect different fungi causing skin fungal infections including yeasts and dermatophytes (Patel et al., 2010 ; Chuang et al., 2007).

\section{AIM OF THE WORK}

The present study is focused on the activity of Moringa olifera seeds on different fungi including dermatophyte, nondermatophyte molds and yeasts, which isolated from human skin and nail fungal infections.

\section{MATERIALS AND METHODS}

\section{Materials}

The plants used in the present investigation were Moringa oleifera Lam. The seeds of Moringa oleifera were obtained from El-kanater El-khiria Horticulture Research Station, Ministry of Agriculture and El-Orman Garden, respectively. 


\title{
1. Preparation of plant extracts
}

$30 \mathrm{~g}$ of collected powdered form of leaves and seeds extracts weighed and extracted with $90 \%$ of methanol, $80 \%$ of petroleum ether, $70 \%$ of ethanol and aqueous (distilled water), made up to $250 \mathrm{ml}$ and purified by soxhlet apparatus with their respective boiling temperature, allow to evaporate and collected in small labelled vials (Balajee el al. 2004).

\section{Phytochemical analysis}

\section{Tannins}

$0.5 \mathrm{~g}$ of the moringa oil was mixed with $20 \mathrm{ml}$ water. The mixture was boiled and filtered. Then few drops of $0.1 \%$ ferric chloride were added. The development of the brownish green or blue-black color confirms the presence of tannins.

\section{Saponins}

$2 \mathrm{~g}$ of moringa oil was mixed and boiled with $20 \mathrm{ml}$ of the water and then filtered. $10 \mathrm{ml}$ of this filtrate was further mixed with $5 \mathrm{ml}$ of distilled water and was shaken vigorously for stable persistent froth. The formation of the froth confirms the presence of the saponins.

\section{Flavonoids}

The known quantity of the oil was taken with the ammonia solution and the concentrated sulfuric acid was added and was allowed to develop the color. Development of yellow color indicated the presence of the flavonoids.

\begin{abstract}
Alkaloids
A $0.5 \mathrm{~g}$ of extract was diluted with $10 \mathrm{ml}$ of acid alcohol, boiled and filtered. Two milliliter of diluted ammonia was added to $5 \mathrm{ml}$ of the filtrate. Five milliliter of chloroform was added and shaken gently to extract the alkaloidal base. The chloroform layer was extracted with $10 \mathrm{ml}$ of acetic acid. This was divided into two portions. Meryer's reagent was added to one portion and Draggendorffs reagent to the other. The formation of a cream (with Meryer's reagent) or reddish brown precipitate (with Draggendorffs reagent) was taken as positive for the presence of alkaloid (Trease and Evans,
\end{abstract} 1983).

\section{Isolation and Identification of fungi from human}

Samples were collected from patient attending to outpatient clinic of Dermatology Zagazig University and private Laboratory for mycological examination. All patients were suspected by dermatologist to have dermatomycosis and have positive direct $\mathrm{KOH}$. From 70 samples collected from skin and nail infection; 20 dermatophyte isolates, 21 non dermatophyte moulds and 25 yeast were isolated.Concerning dermatophyte which isolated only from skin infections they were identified by macromorphological and micromorphological characters as well as cultures on rice grains, BCP medium and MHB medium into: Microsporum canis, Trichophyton violaceum, Trichophyton mentagrophytes and Trichophyton rubrum. M. canis and T.violaceum were isolated as the most predominant cause of Tinea capitis the result which coincide with previous works done by El Fangary et al (2011) and Soliman et al (2013). From Tinea corporis and tinea pedis T. rubrum, T.mentagrophytes, M. canis and T. violaceum were the most dermaophytes isolated the results which in agreement with El Fangary et al., (2011) and Taha et al., (2013).

In the present work 21 non dermatophytes moulds $(42 \%)$ were isolated from cases of Onychomycosis, the identification of non dermatophytes moulds isolated from Onychomycosis were done according macromorphological and micromorphological characters revealed the isolation of Aspergillus niger (12\%), Aspergillus nidulans 14\%, Aspergillus flavus (10\%), Fusarium oxysporum (4\%) and one Scytalidium dimidiatum 2\%..The high incidence of non dermatophytes molds were Aspergillus species the result which was also observed by El Khafagy et al. (2002) and Taha (2011). Yeast isolates which obtained from Onychomycosis were identified by macroscopic on CCA and microscopic on corn meal agar besides and macromorphological on SDA as well as micromorphological after Gram stain.

\section{Agar well diffusion susceptibility test determination of MIC (Gergova et al., 2005).}

Petridishes were prepared with Müller Hinton for yeast and with potato dextrose for dermatophyte and non dermatophyte moulds. Five welles $(6 \mathrm{~mm}$ in diamater were then cut for agar). $100 \mathrm{Mu}$ of ethanolic Moringa oleifera L. extract in concentration of $(100 \%, 75 \%, 50 \%, 25 \%) .0 \%$ it was used as control where added into the wells after incubation for 3-5 days for yeasts and 5:8 days for non dermatophyte and $8: 10$ days for dermatophyte dishes were examined for zones of growth inhibition the result were reported.

Agar dilution method (Irith et al., 2008)

For MIC determination ethanol extract of Moringa oleifera L. A serial dilution was done with in $(100 \%, 75 \%, 50 \%$ and $25 \%$ ). One volume of the solution was added to 100 vol. of Müller Hinton media for yeast and PDA for other moulds. mixed thoroughly and the mixture poured in the sterilized petri dishes after solidification one loop of the tests strain of fungi was inoculated in the surface of the medium. Control was done by inoculated tests strains on media free from the extract. The plates were incubated for 48 hours for yeast, 7 days for non dermatophyte moulds and 15 days for 
dermatophyte. After confirming that sufficiency growth was obtained on the growth control plate end point were read. The lowest drug concentration of the plate showing no colony formation was recorded as MIC.

\section{Transmission electron microscope:}

In this experiment, transmission electron microscope was applied to detect the effect of Moringa oleifera seed extract in the conidia cell wall and mycelia of A. flavus at Petroleum ether extract and ethanol extract compared with control (without Moringa oleifera seed extract). A small portion of the fungal mat was fixed at room temperature in $2 \%(\mathrm{v} / \mathrm{v})$ glutaraldehyde mixed with potassium in $2 \%$ (w/v) osmium tetraoxide buffered in $0.005 \mathrm{M}$ sodium cacodylate at $\mathrm{pH} 6.5$ for $40 \mathrm{~min}$. After fixation, the material was washed overnight in the appropriate buffer, dehydrated at room temperature in acetone, and embedded overnight at $65^{\circ} \mathrm{C}$ in low viscosity epoxy resin (Spuur, 1969). At these conditions, the material was polymerized; ultrathin sections were cut by glass knives of an ULKD ultramicrotome. Sections were collected each on stabilized copper grids, stained with lead citrate and examined in a GOL 100 CX electron microscope. This method was carried out according to the instructions of Ellis and Griffiths (1974) and was applied in T.E.M. unit in Faculty of Agriculture, Cairo University.

\section{RESULTS AND DISCUSSION}

\section{Preliminary phytochemical analysis of petroleum extract $(A)$ and ethanol extract $(B)$}

The presence of Alkaloids, Flavonoids, Saponins and tannins were observed in (A, B) extracts in Table (1). Alkaloids are present in the ethanol extract (B) and absent in Petroleum ether extract (A). Flavonoids are present in (A) and (B). Saponins are present in (B) and absent in (A). Tannins are present in (A) and (B).

These results reavealed that ethanol extract contain phytochemical charaxteristics more than the other extracts .

Table (1) : preliminary phytochemical analysis of petroleum extract $(\mathrm{A})$ and ethanol extract $(\mathrm{B})$

\begin{tabular}{|l|c|c|}
\hline Tests & A & B \\
\hline Alkaloids & - & + \\
\hline Flavonoids & + & + \\
\hline Saponins & - & + \\
\hline Tannins & + & + \\
\hline
\end{tabular}

$\mathrm{A}=$ Petroleum ether extract, $\mathrm{B}=$ Ethanol extract

$(+)=$ presence of compound, $(-)=$ absence of compound .

\section{Antifungal Activity}

In the present work antifungal activity of Moringa oleifera seeds was done using ethanol extract as (EME) as well as petroleum ether extract. Susceptality test for Moringa olifera seeds extracts against dermatophytes (M. canis, T.mentagrophytes and T.rubrum). non dermatophytes moulds (A. niger, A. nidulens A. flavus, and F. oxysporum) as well as yeast (C. albicans. C.purpalers, C. Kirusei and R. mucilagenan) were done by well diffusion method comparing with itraconazole disc.

Table (2): Inhibition zone of EME, PME and itraconzole on different fungi/mm

\begin{tabular}{|l|c|c|c|}
\hline Fungi & EME & PME & itraconzole \\
\hline Dermatophytes & & & \\
M. canis & 28 & 18 & 30 \\
T. mentagrophytes & 35 & 10 & 28 \\
T. rubrum & 30 & 12 & 25 \\
\hline Non dermatophyte moulds & 20 & & \\
Aspergillus niger & 17 & 7 & 22 \\
Aspergillus nidulans & 15 & 5 & 18 \\
Aspergillus flavus & 20 & 10 & 20 \\
Fusarium oxysporum & & & 15 \\
\hline Yeast & $18-30$ & $0-10$ & 28 \\
\hline Candida albicans & 30 & 10 & 25 \\
Candida parapsilosis & 10 & 10 & 24 \\
Candida krusei, & 35 & 15 & 30 \\
Rhodotorula mucilaginosa & \multicolumn{2}{|l}{} \\
\hline
\end{tabular}


The results reversed moderate to been effect of EMG against NDM and yeast comparing to itraconzole while inhibition varies from 15-22 $\mathrm{mm}$ as the other hand yeast inhibition zone done by EME reversed effective against C. albicans (18-30 $\mathrm{mm})$ C. parapsilosis $(30 \mathrm{~mm})$ and $35 \mathrm{~mm}$ inhibition zone against R. muciloginosa. C. krusei only showed been inhibition zone $(10 \mathrm{~mm})$

For all yeast PME showed been effective rang from 10-15 mm. Control disk of itraconzole showed been 24-30 mm for all yeasts (table 2).

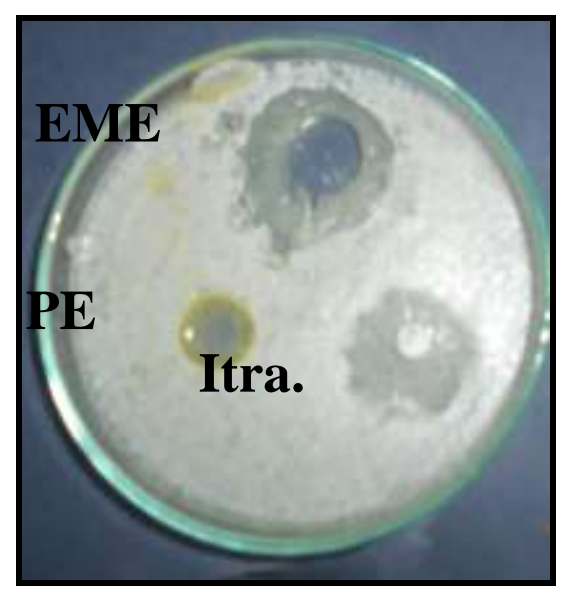

Figure 1: Antifungal effect of EME and PE compared to Itraconazole on candida albicans

MIC of ethanolic extract of moringa oleifera against dermatophyte reversed strongest antifungal activity for $\mathrm{T}$. mentagrophytes $100 \%$ and T. rubrum $25 \%$. While Mic for M.canis moderate activity showed been $50 \%$. Ethanolic extract of moringa oleifera against non dermatophyte mould showed been inhibition where MIC ranging from 50-75\%.

On the other hand MIC of ethanolic extract of moringa oleifera against yeast were variant with increase inhibition against C. albicans, C. parapsilosis and R. mucilaginosa $25 \%$ and moderate inhibition effect by C. krusei where MIC was $50 \%$.

Table (3): MIC of ethanol extract of Moringa oleifera L. against dermatophyte

\begin{tabular}{|l|l|l|l|}
\hline Concentration & M. canis & T. mentagrophytes & T. rubrum \\
\hline $75 \%$ & - & - & - \\
\hline $50 \%$ & - & - & - \\
\hline $25 \%$ & + & - & - \\
\hline $10 \%$ & + & - & + \\
\hline
\end{tabular}

+ indicates growth of dermatophyte - indicates inhibition of growth

Table (4): MIC of ethanol extract of Moringa oleifera L. against Non dermatophyte moulds

\begin{tabular}{|l|l|l|l|l|}
\hline Concentration & A. niger & A. nidulans & A. flavus & F. oxysporum \\
\hline $75 \%$ & - & - & - & - \\
\hline $50 \%$ & - & + & + & - \\
\hline $25 \%$ & + & + & + & + \\
\hline $10 \%$ & + & + & + & + \\
\hline
\end{tabular}

Table (5): MIC of ethanol extract of Moringa oleifera L. against yeast

\begin{tabular}{|l|c|c|c|c|}
\hline Concentration & C. albicans & C. parapsilosis & C. krusei & R. muciloginosa \\
\hline $75 \%$ & - & - & - & - \\
\hline $50 \%$ & - & - & - & - \\
\hline $25 \%$ & - & - & + & - \\
\hline $10 \%$ & + & + & + & + \\
\hline $5 \%$ & + & - & - & + \\
\hline
\end{tabular}

The current work concerning the antifungal activity of EMO on dermatophyte, non-dermatophyte molds and yeasts are in agreement with Riad (2014). 


\section{Effect of Moringa oleifera seed extract on the cell wall of Aspergillus flavus by transmission electron microscope:}

From the table (2), Inhibition zone of EME, PME on different fungi we found that the antifungal activity of Aspergillus flavus in PME gave $5 \mathrm{~mm}$ which is lowest effect on different fungi and EME gave $15 \mathrm{~mm}$, thus we had done a comparison between the effect of EME, PME on the cell wall of Aspergillus flavus by transmission electron microscope.

Examining A. flavus morphology in the control sample indicated normal structure for mycelia and conidia of the fungus (Fig. 2 A). Petroleum ether extract of Moringa oleifera seed had affects on cell wall; forming some external protrusion, in both conidia and mycelium, degenerated intercellular septa in mycelium with torned cell wall in conidia and cause irregular cell wall (Fig. 2 B). A. flavus treated with ethanol extract of Moringa oleifera highly affected the fungus morphology and led to irregular cell shape with destroyed cell wall and shrinkage of cell cavity (Fig. 2 C).
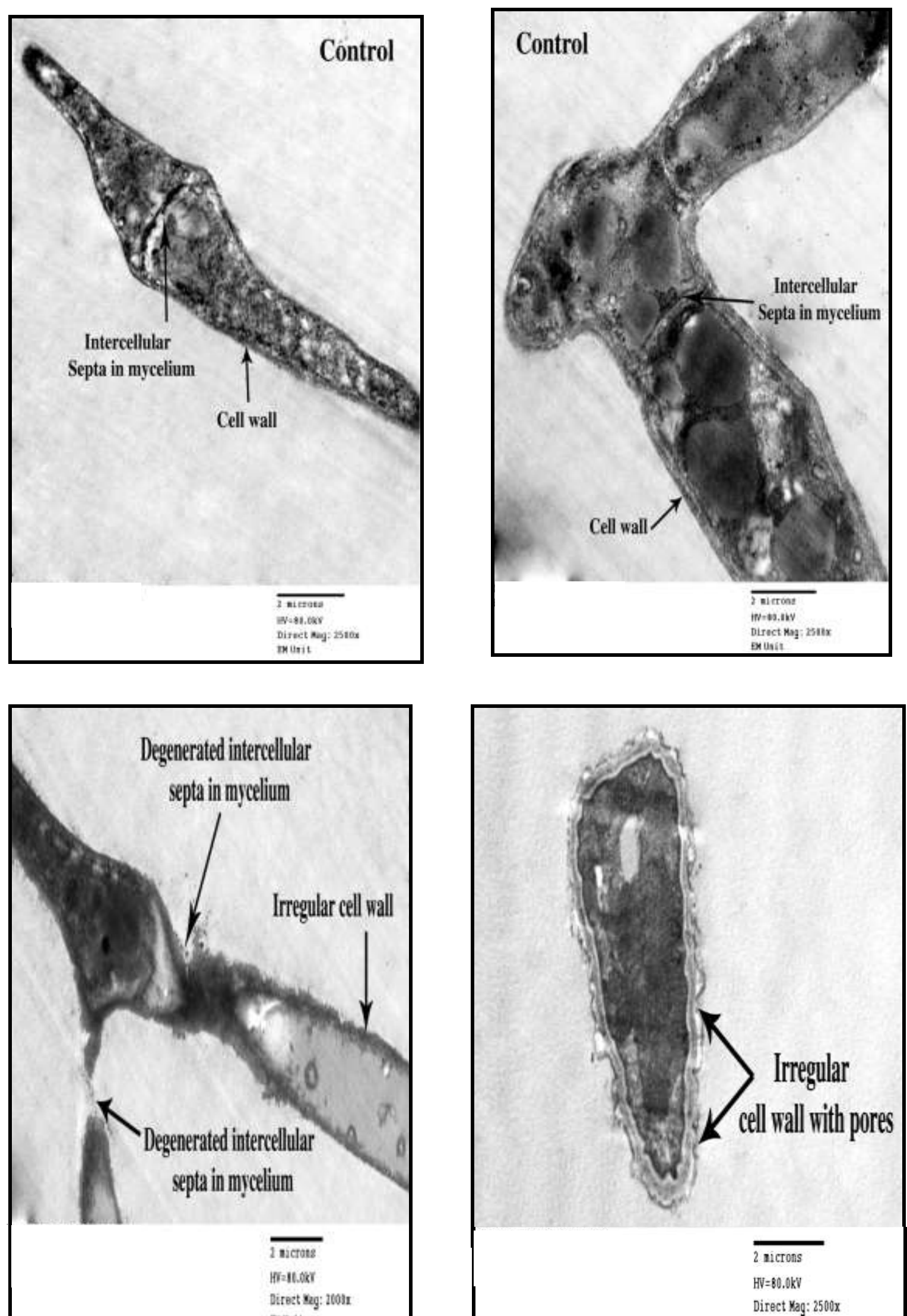

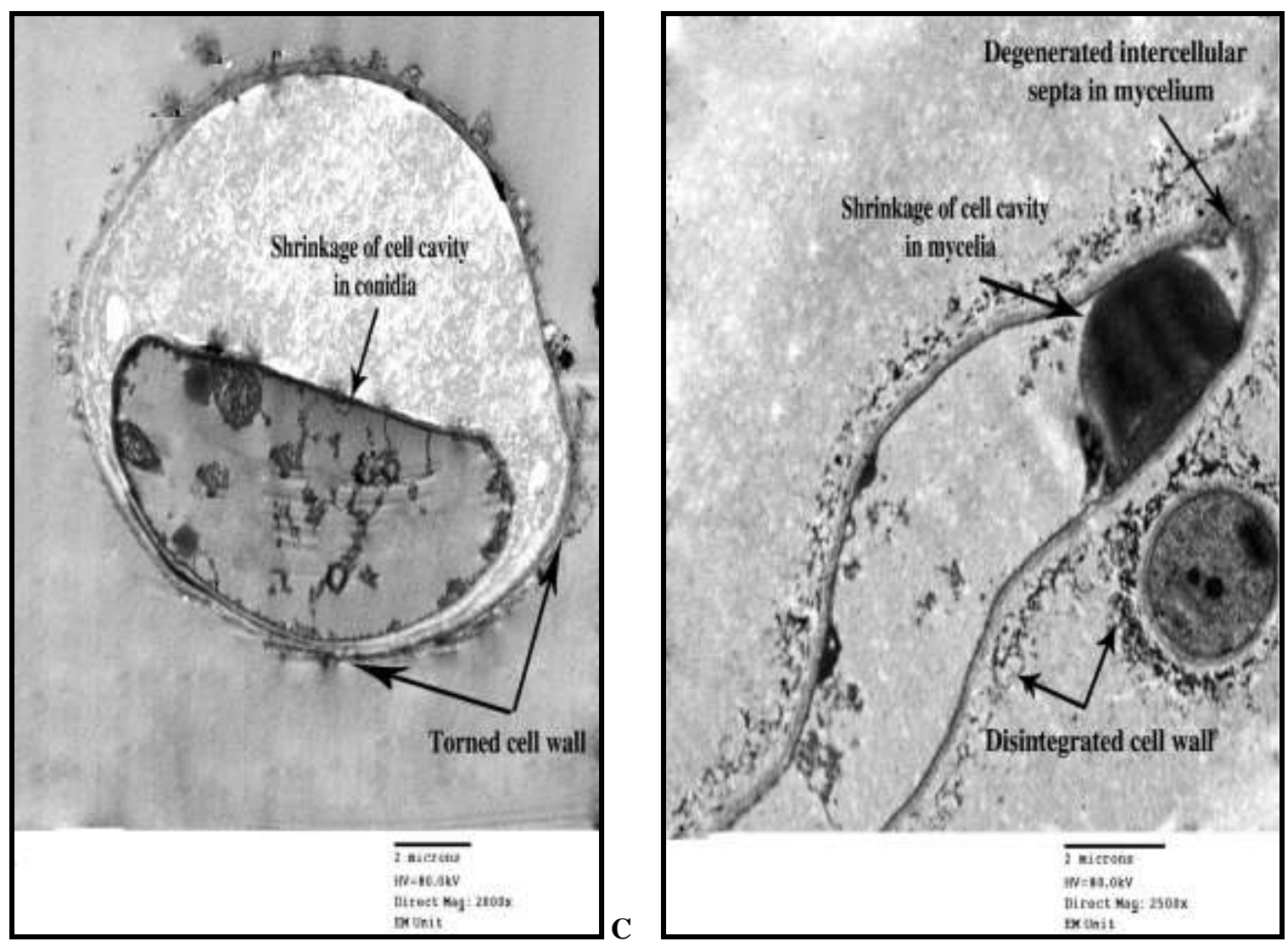

Figure 2: Control (A), effect of petroleum ether (B) and ethanol extract (C) of Moringa oleifera seeds on the cell wall of Aspergillus flavus by transmission electron microscope

Changes in cell wall of fungus; external, disintegration and irregular cell wall was found under TEM treated with Petroleum ether extract and ethanol extract of Moringa oleifera seeds. Also there are some deformation inside cell and shrinkage of cell cavity this may be due to presence of antioxidant properties of tested extract which are effective superoxide antioxidants with ability to inhibit mycelial growth by reacting with cell wall components (Chandrasekaran et al., 2011). From research findings, it has been noted that n-Hexadecanoic acid, Octadecanoic acid and Tetradecanoic acid found on Moringa oleifera seed extracts may have target site on the cell wall of this fungus. The mode of action may possibly be by attack on the sugar residues on the cell wall of fungal species according to (Rahuman et al., 2000). Antifungal activity of Moringa oleifera seed could be correlated to its scavenging action on superoxide and hydroxyl radical which could be part of cellular metabolism of the enteropathogens (Mahmood et al., 2009).

\section{CONCLUSION}

Dermatophytes, non dermatophyte fungi are the cause of human skin and nail infections, While the dermatophytes are predominant in skin infections non dermatophyte fungi are more common in onychomycosis ( nail infections).

While macromorphological and micromorphological as well as cultivation on differential media identified all isolated molds (derematophyte and non dermatophye molds), cultivation on corn meal media and chromogenic Candida agar could identified isolated yeasts.

EME is have more antifungal activity than PME on all tested fungi. Dermatophytes are the more sensitive fungi for the activity of Moringa olifera extracts. Except Candida krusei all tested yeasts were sensitive for EME. Moringa olifera extracts is active against dermatophyte,non-dermatophyte and yeasts, therefore.it could be used as an alternate source for the treatment skin diseases caused by fungi. 


\section{REFERENCES}

- Balajee et. al., (2004): The ethanolic extracts of leaves of o.sanctum against Cryptococcus neoforms. (1):0968157

- Chandrasekaran M., Senthilkumar A. and Venkatesalu, V. (2011): Antibacterial and antifungal efficacy of fatty acid methyl esters from leaves of Sesuvium portulacastrum L. Eur. Rev. Med. Pharmcol. Sci.; 15: 775-780.

- Chuang P, Lee C, Chou J et al. (2007): Anti-fungal activity of crude extracts and essential oil of Moringa oleifera Lam. Bioresour Technol.;98:232-236.

- Ekor M (2014): The growing use of herbal medicines: issues relating to adverse reactions and challenges in monitoring safety. Front Pharmacol.; 10 (4):177

- El Fangary M, Saudy W, Nabil T et al. (2011): Prevalence of dermatophytes, yeasts, non dermatophyte moulds isolated from skin, hair, and nail fungal infections in the 6th October City-Giza Egypt. Egypt J Dermatol Venerol.; 31 :5-10.

- Ellis P and Griffiths D (1974): The locat ion and analysis of melanins in the cell walls of some soil fungi. Can. J. Microbiol., 20(10): 1379-1386.

- Gergova G, Boyanova L, Nikolov R et al. (2005): Activity of Bulgarian propolis against 94 Helicobacter pylori strains in vitro by agar-well diffusion, agar dilution and disc diffusion methods. J Med Microbiol.;54(Pt 5):4813.

- Irith W, Kai H and Robert E (2008): Agar and broth dilution methods to determine the minimal inhibitory concentration (MIC) of antimicrobial substances. Nature Rotocols, VOL.3 NO.2 : 163-175.

- Kayode R and Afolayan A (2015): Cytotoxicity and effect of extraction methods on the chemical composition of essential oils of Moringa oleifera seeds. J Zhejiang Univ-Sci B (Biomed \& Biotechnol);16(4):680-689

- Khafagy M, El-Wahas A, Eid F et al. (2002): Synthesis of halogen derivatives of benzo[h]chromene and benzo[a]anthracene with promising antimicrobial activities, Farmaco, 57(9):715-22.

- Mahmood A., Ahmed R. and Kosar S. (2009): Phytochemical screening and biological activities of the oil components of Prunus domestica Linn. J. Saudi Chem. Soc.; 13, 273-277.

- Pan SY, Gao SH, Zhou SF et al. (2012): New perspectives on complementary and alternative medicine: an overview and alternative therapy. Alternative Therapies in Health and Medicine.;18(4):20-36.

- Patel P, Summaiya M, Disha P et al. (2010): A study of superficial mycosis in south Gujarat region. National Journal of Community Medicine, 1 (2): 85-88.

- Rahuman A., Gopalakrishnan G., Ghouse B. et al. (2000): Arumugam, S.; Himalayan, B. Effect of Feronia limonia on mosquito larvae. Fitoterapia; 71, 553-555.

- Ramachandran C, Peter K and Gopalakrishnana P (1980): Drumstick (Moringa oleifera) : A multipurpose Indian vegetable. Econ. Bot. 34: 276-283.

- Riad S, El-Mohamedy R and Aboelfetoh M. (2014): Antifungal activity of Moringa oleifera oil and seed extract against some plant pathogenic fungi. Middle East Journal of Agriculture Research, 3(2): 242-249.

- Soliman A, Taha M and Samia A (2013): Correlation between phenotypic and genotypic identification of trichophyton violaceum. MD thesis, Zagazig university.

- Spuur A (1969): A low viscosity epoxy resin embedding medium $f$ or electron microscopy. J. Ultrastruct. Res., 26 (1): $31-41$

- Tabassum N and Vidyasagar G (2013): Antifungal Investigations On Plant Essential Oils. A Review. International Journal of Pharmacy and Pharmaceutical Sciences, 5 (2): 19-28

- Taha M (2011): Dermatophytosis. In Taha M: Medical mycology. Atlas of medically important fungi and dermatomycosis. $l^{\text {st }}$ edition. Mecca Printing House. Cairo-Egypt, p238-252.

- Taha M, El fangary M and Saudy W (2013): MHB: a new medium for isolation and identification of dermatophytes. J E W D S; 10 (3): 172-176.

- Trease, G.E. and Evans, W.C. (1983): Textbook of pharmacognosy. 12th Edition, Tindall and Co., London, 343383. 